\title{
The Clinical Research Progress of Vertebral Artery Dominance and Posterior Circulation Ischemic Stroke
}

\author{
Yan Sun ${ }^{a}$ Yan-Min Shi ${ }^{b}$ Ping $X u^{a}$ \\ ${ }^{a}$ Department of Neurology, The Second Hospital of Jilin University, Changchun, China; ${ }^{b}$ Department of Medical \\ Examination Center, The Second Hospital of Jilin University, Changchun, China
}

\section{Keywords}

Vertebral artery dominance - Vertebral artery dysplasia .

Posterior circulation ischemic stroke $\cdot$ Hemodynamics

\begin{abstract}
Background: The diameters of the vertebral arteries on both sides are usually asymmetrical. Vertebral artery dominance (VAD) and congenital vertebral artery dysplasia are derived from the asymmetry of the vertebral artery diameters on both sides. They are usually regarded as normal congenital vascular variations and have no practical clinical significance. Summary: Recently, several clinical studies have found that VAD is a risk factor for posterior circulation ischemic stroke (PCIS). The existence of VAD is clinically significant for the occurrence of PCIS, and it is recognized by an increasing number of clinicians. Key Messages: This article reviews the frequency and evaluation methods of VAD, its correlation with PCIS, and its mechanism.

(C) 2022 S. Karger AG, Basel
\end{abstract}

\section{Introduction}

Traditionally, vertebral artery dominance (VAD) is considered a normal congenital vascular variation of the vertebral arteries (VAs). It usually refers to the obvious asymmetry of the VA diameters on both sides. The side with the larger pipe diameter is the dominant side, and the side with the smaller pipe diameter is the nondominant side [1]. Therefore, the correlation between the occurrence and the development direction of VAD and cerebrovascular diseases has been ignored. However, there is increasing evidence [2-4] that VAD is associated with posterior circulation ischemic stroke (PCIS). The frequency of VAD is higher in patients with PCIS than in those with anterior circulation ischemic stroke and is associated with basilar artery (BA) curvature. However, there are few studies on the hemodynamics of the junction between VAD and the vertebrobasilar artery and the presence of VAD and its role in the occurrence of PCIS.

\section{Frequency of VAD}

The VAs originate from the subclavian artery, and the two VAs converge to form the BA at the lower margin of the junction of the medulla oblongata and the pons. The diameter of the VA tube on the left is usually slightly thicker than that on the right. This may be related to the fact that the left VA is the secondary branch of the aortic arch while the right VA is the tertiary branch of the aortic arch; therefore, the left VA is subjected to higher shear force during development $[1,3]$. VAD is a common congenital variation of the VAs. About $50 \%$ of people have a dominant VA on the left side, and approximately $25 \%$ of people have essentially equal-sized bilateral VA tubes [2]. karger@karger.com www.karger.com/ced
(C) 2022 S. Karger AG, Basel

Karger ${ }^{\prime}=$
Correspondence to:

Ping Xu, xuping_12@outlook.com 


\section{VAD Evaluation Method}

Through color Doppler ultrasound, magnetic resonance angiography (MRA), computed tomography angiography (CTA), and digital subtraction angiography (DSA), we can evaluate the origin, course, and variation of the VA diameter $[5,6]$.

\section{Color Doppler Ultrasound}

As a noninvasive examination method, color Doppler ultrasound can clearly display the wall structure and lumen section images of the examined vessel, provide hemodynamic parameters, quickly evaluate the situation of extracranial artery occlusion, and guide clinical decisionmaking $[7,8]$. However, the examination analysis may be affected by the receptor location and individual differences and largely depends on the operating level of the examiner. Moreover, because of technical limitations, the VA suboccipital segment and intracranial segment cannot be displayed. However, due to its simplicity, noninvasiveness, low cost, ease of use, and wide availability, it is currently the preferred method commonly used to evaluate the extracranial arteries [9].

\section{MRA and CTA}

MRA is a noninvasive vascular imaging method that uses magnetic sensitivity to flowing fluid to generate images of occluded vessels with high accuracy [6]. CTA is an important examination method, providing a reliable basis for preoperative evaluation and the diagnosis of cerebrovascular diseases [10]. However, the cost of CTA is high, the amount of radiation is large, and those allergic to contrast agents should not undergo this examination. In addition, MRA and CTA only display the blood flow in the vascular lumen and not in the vascular wall structure. When the thickening of the VA wall causes diffuse stenosis in the vascular lumen, the diagnosis of VAD will appear as a false phenomenon.

\section{Digital Subtraction Angiography}

DSA [11] is the gold standard for vascular diagnosis and measurement, and interventional therapy can be performed simultaneously. However, it is an invasive examination, with various complications and high cost, and it cannot show the bone structure around blood vessels, so it is not readily accepted by patients. In addition, due to angle limitations, the stenosis rate may be overestimated or underestimated during measurement.

\section{Clinical Study of VAD and PCIS}

Historically, VAD was considered by most clinicians to be a normal congenital vascular anomaly or a clinically insignificant finding unless symptoms of insufficient blood supply to the vertebrobasilar system occurred. Recently, however, increasing clinical attention has been paid to the close relationship between VAD and PCIS, and studies have considered VAD as a risk factor for PCIS [12]. Zhu et al. [3] found that the frequency of posterior circulation cerebral infarction in the VAD group (46.2\%) was higher than that in the control group (20.6\%) with a statistically significant difference. Additionally, the frequency of posterior inferior cerebellar artery infarction $(11.5 \%$ vs. $1.5 \%, p=0.016)$ and BA infarction $(20.5 \%$ vs. $7.4 \%, p=0.024$ ) was higher in the VAD group than that in the control group. These findings suggested that VAD was related to PCIS. In a study on acute pontine infarction [13], affected patients were divided into a BA bending group and a BA nonbending group. Patients with BA bending that demonstrated symptoms of dizziness or vertigo but did not develop cerebral infarction were the control group. In the bending group, nonbending group, and control group, VAD proved to be a significant difference. In all three groups, multivariate analysis showed that a grade 3 bending length $(3.77-7.25 \mathrm{~mm})$ was an independent risk factor for pontine infarction. Both bending length and diameter were positively correlated with the risk of pontine infarction, both of which were statistically significant. The co-existence of BA bending and vascular risk factors should be of great concern because of the increased risk of pontine infarction. There are few clinical studies on VAD and PCIS, and whether VAD is an independent risk factor for PCIS patients requires more and larger multicenter studies in the future.

\section{The Mechanism of VAD and PCIS}

Increasing attention has been given to the theory that VAD causes BA bending, and its clinical significance is that it increases the possibility of PCIS. One study found that [14] infarction in the BA region mostly occurred on the opposite side of BA bending, while infarction in the blood supply area of the posterior inferior cerebellar artery mostly occurred on the nondominant side of the VA. These results suggest that the location of the infarction is closely related to BA bending or VAD. Recent studies [15] also support that BA bending depends on the dominance of the VA, and most subjects have an inverse relationship 
between the dominance of the VA and $\mathrm{BA}$ bending (71.3\%).

The asymmetric flow pattern at the vertebrobasilar junction may be an important mechanical force of BA bending $[13,16]$. Blood flow from unequal VAs to the merged BA shifts the BA flow curve to the weaker side of the VAs. VAD can significantly change the hemodynamic symmetry of the vertebrobasilar junction, resulting in BA bending. The greater the difference in the VA diameter between the two sides, the greater the BA bending will be (likely caused by a chronic process caused by asymmetric VA flow). Such deformation tends to lead to atherosclerosis; this promotes the thickening and loss of elasticity of the arterial wall and ultimately leads to the elongation and dilation of the artery $[3,17]$. The posterior inferior cerebellar artery is a branch of the VA and has no other collateral circulation. The interaction between low blood flow and impaired dynamic blood flow regulation may lead to posterior circulation ischemia and eventually lead to infarction in the blood-supplying region of the posterior inferior cerebellar artery $[18,19]$.

Available evidence suggests that dysfunction increases the vascular risk of posterior circulation ischemic events and provides a possible physiological mechanism $[2,19]$. When the blood flow of one side of the vertebral artery decreases, the blood flow of the contralateral vertebral artery will be increased compensatorily to provide sufficient blood flow to ensure sufficient cerebral tissue perfusion in the posterior circulation. In a healthy human body, the compensatory mechanism of blood works, and clinical symptoms do not appear. The compensatory ability of the human body decreases with age, and the compensatory ability is limited. If it coexists with other risk factors, it may greatly increase the risk of posterior circulation ischemic events. VAD plays an important role in the formation of basilar artery curvature and posterior circulation ischemia. The remodeling ability of the nondominant vertebral artery is reduced. According to Bernoulli's principle, small-caliber blood vessels are more likely to collapse due to greater pressure. The non-dominant vertebral artery is likely to cause infarction in the ipsilateral posterior inferior cerebellar artery area due to the decrease of blood flow into the skull $[4,20,21]$. Longterm asymmetric vertebral artery blood flow will cause the basilar artery to bend further, and the curved blood vessel will aggravate atherosclerosis, which will cause vascular stenosis, occlusion and a series of pathophysiological changes. The interaction between hemodynamic imbalance and impaired dynamic blood flow regulation can lead to ischemia in the posterior circulation, and dysfunc-

New Progress in Vertebral Artery

Dominance tion of dynamic blood flow regulation may also increase the risk of thrombosis. In short, more research is needed to clarify the characteristics of the hemodynamic changes of the vertebral artery dominance phenomenon.

\section{Outlook}

Clinicians and radiologists primarily focus on vascular stenosis and occlusion and often ignore the existence of $\mathrm{VAD}$ in the treatment and prevention of cerebrovascular diseases. Clinicians need to pay attention to the clinical significance of VAD. Currently, a standard definition of VAD has not been determined, and more clinical studies are needed to determine a definition that links VAD with PCIS and is of clinical significance. Asymptomatic people with congenital mutations should assess the potential risk of ischemic events, individualized treatment, or simple follow-up. In addition, early primary and secondary prevention for high-risk groups is essential.

\section{Acknowledgments}

We are particularly grateful to all the people who have helped us with our article.

\section{Conflict of Interest Statement}

The authors declare that they have no competing interests.

\section{Funding Sources}

No external funding was received to conduct this study.

\section{Author Contributions}

Conception and design of the research: Yan Sun and Ping Xu. Acquisition of data: Ping Xu and Yan-Min Shi. Analysis and interpretation of the data: Yan Sun and Yan-Min Shi. Statistical analysis: Ping Xu. Obtaining financing: none. Writing of the manuscript: Yan Sun. Critical revision of the manuscript for intellectual content: Yan Sun and Ping Xu. All authors read and approved the final draft.

\section{Data Availability Statement}

The transcripts from which this manuscript was developed are available on request from the corresponding author. 


\section{References}

1 Jeng JS, Yip PK. Evaluation of vertebral artery hypoplasia and asymmetry by color-coded duplex ultrasonography. Ultrasound Med Biol. 2004;30(5):605-9.

2 Katsanos AH, Kosmidou M, Kyritsis AP, Giannopoulos S. Is vertebral artery hypoplasia a predisposing factor for posterior circulation cerebral ischemic events? A comprehensive review. Eur Neurol. 2013;70(1-2):78-83.

3 Zhu W, Wang YF, Dong XF, Feng HX, Zhao HQ, Liu CF. Study on the correlation of vertebral artery dominance, basilar artery curvature and posterior circulation infarction. Acta Neurol Belg. 2016;116(3):287-93.

4 Hu XY, Li ZX, Liu HQ, Zhang M, Wei ML, Fang S, et al. Relationship between vertebral artery hypoplasia and posterior circulation stroke in Chinese patients. Neuroradiology. 2013;55(3):291-5.

5 Peterson C, Phillips L, Linden A, Hsu W. Vertebral artery hypoplasia: prevalence and reliability of identifying and grading its severity on magnetic resonance imaging scans. J Manipulative Physiol Ther. 2010;33(3):207-11.

6 Baik J, Baek HJ, Shin HS, Choi KH. Duplication of the right vertebral artery: MRA findings and review of the literature. Springerplus. 2016;5(1):1123

7 Accorsi F. Color Doppler of the extracranial and intracranial arteries in the acute phase of cerebral ischemia. J Ultrasound. 2013;16(4): $187-93$.
8 Nicolau C, Gilabert R, Chamorro A, Vázquez F, Bargalló N, Brú C. Doppler sonography of the intertransverse segment of the vertebral artery. J Ultrasound Med. 2000;19(1):47-53.

9 Chi HY, Hsu CF, Chen AC, Su CH, Hu HH, $\mathrm{Fu}$ WM. Extracranial and intracranial ultrasonographic findings in posterior circulation infarction. J Ultrasound Med. 2018;37(7): 1605-10.

10 Kim C, Sohn JH, Choi HC. Are the anomalous vertebral arteries more hypoplastic?: retrospective linear mixed model approach. BMC Neurol. 2017;17(1):168.

11 Zhao H, Wang J, Liu X, Zhao X, Hippe DS, Cao Y, et al. Assessment of carotid artery atherosclerotic disease by using three-dimensional fast black-blood MR imaging: comparison with DSA. Radiology. 2015;274(2):50816.

12 Meng X, Ding W, Wu X, Di P. Clinical investigation and characterization of vertebrobasilar dolichoectasia and vertebral artery dominance. Discov Med. 2018;25(138):151-8.

13 Zhang DP, Zhang SL, Zhang JW, Zhang HT, Fu SQ, Yu M, et al. Basilar artery bending length, vascular risk factors, and pontine infarction. J Neurol Sci. 2014;338(1-2):142-7.

14 Park JH, Kim JM, Roh JK. Hypoplastic vertebral artery: frequency and associations with ischaemic stroke territory. J Neurol Neurosurg Psychiatry. 2007;78(9):954-8.
15 Ngo MT, Kwak HS, Chung GH. Change in basilar artery length and bending according to aging and vertebral artery dominance: a longitudinal study. Sci Rep. 2020;10(1):8904. http: //dx.doi.org/10.1038/s41598-02065682-x.

16 Cunningham KS, Gotlieb AI. Change in basilar artery length and bending according to aging and vertebral artery dominance: a longitudinal study. Sci Rep. 2005;85(7):942.

17 Kumral E, Kisabay A, Ataç C, Kaya C, Calli C. The mechanism of ischemic stroke in patients with dolichoectatic basilar artery. Eur J Neurol. 2005;12(6):437-44.

18 Thierfelder KM, Baumann AB, Sommer WH, Armbruster M, Opherk C, Janssen H, et al. Vertebral artery hypoplasia: frequency and effect on cerebellar blood flow characteristics. Stroke. 2014;45(5):1363-8.

19 Chen YY, Chao AC, Hsu HY, Chung CP, Hu $\mathrm{HH}$. Vertebral artery hypoplasia is associated with a decrease in net vertebral flow volume. Ultrasound Med Biol. 2010;36(1):38-43.

20 Chuang YM, Huang YC, $\mathrm{Hu} \mathrm{HH}$, Yang CY. Toward a further elucidation: role of vertebral artery hypoplasia in acute ischemic stroke. Eur Neurol. 2006;55:193-7.

21 Sato K, Yoneya M, Otsuki A, Sadamoto T, Ogoh S. Anatomical vertebral artery hypoplasia and insufficiency impairs dynamic blood flow regulation. Clin Physiol Funct Imaging. 2015;35(6):485-9. 